\title{
Comprehensive modeling of prompt fission neutrons and $\gamma$ rays in the spontaneous fission of ${ }^{252} \mathrm{Cf}$
}

\author{
Patrick Talou ${ }^{\mathrm{a}}$, Ionel Stetcu, and Toshihiko Kawano \\ Nuclear Physics Group, Theoretical Division, Los Alamos National Laboratory, Los Alamos, NM 87545, USA
}

\begin{abstract}
We present a comprehensive set of calculations performed with the Monte Carlo Hauser-Feshbach code CGMF of the prompt fission neutrons and $\gamma$ rays emitted in the spontaneous fission of Cf-252. This reaction has been studied in depth over the years and provides an almost perfect test for the assumptions, parameters and output of the CGMF code. Here we present results for prompt neutron observables beyond the ubiquitous average prompt fission neutron spectrum and multiplicity. In particular, we compare CGMF calculations to experimental data on neutron data per fragment mass split, neutron-light fragment and neutron-neutron angular distributions, and on the time dependence of the average prompt $\gamma$-ray multiplicity. Finally, we briefly discuss the recent integration of CGMF into the MCNP6.2 transport code.
\end{abstract}

\section{Introduction}

The study of prompt fission neutrons and $\gamma$ rays has seen a resurgence in the last few years due in part to the development of event-by-event fission fragment evaporation codes [1-4] and to the design of experiments aimed at measuring correlations between those particles. While nuclear data evaluations have been mostly confined to the estimation of an average prompt fission neutron spectrum (PFNS) $\langle\chi\rangle$, average prompt fission neutron multiplicity (PFNM) $\bar{v}$, and, for a few nuclei, the same quantities for the $\gamma$ rays, these new tools can provide significantly more data, thereby placing indirect constraints on the evaluated data themselves. Indeed, they can be used to compute the complete kinematics of emission of prompt neutrons and $\gamma$ rays, on an eventby-event basis. The type of data that can be extracted from such simulations is limitless, and many interesting correlations can be inferred between the emitted particles in terms of multiplicity, energy and direction of emission.

The spontaneous fission of ${ }^{252} \mathrm{Cf}$ provides a unique benchmark for such simulations. It has been studied by many experimental groups, providing many data sets, usually with high statistics, and unique distribution and correlation measurements have been reported. Similar but only very partial data can be found for other fission reactions, e.g., for ${ }^{235} \mathrm{U}$ and ${ }^{239} \mathrm{Pu}$ thermal neutron-induced fission reactions. Higher-energy data are scarce. The vast amount of experimental data available for ${ }^{252} \mathrm{Cf}$ (sf) provides a very stringent test for some of the model assumptions made in statistical evaporation codes. This is the object of this short paper.

\section{Theoretical models}

We provide here a very brief description of the models used, and more information can be found in CGMF User

\footnotetext{
a e-mail: talou@lanl.gov
}

Manual [5]. In our approach, the emission of prompt fission neutrons and $\gamma$ rays happens solely from fully accelerated fission fragments. We therefore assume that no neutron can be emitted prior to scission, except in the case of neutron-induced multi-chance fission. We also limit ourselves to binary fission events, in which only two complementary fragments are emitted in opposite directions. Once separated, the fission fragments quickly recover their ground-state shapes and the extra deformation energy due to their elongation at scission gets converted into intrinsic excitation energy. This energy is eventually released by the emission of prompt neutrons and $\gamma$ rays, in competition. The statistical HauserFeshbach model is then used to describe this decay, with emission probabilities obtained from the models described below.

The prompt neutron emission probability is governed by the product of the neutron transmission coefficients and the level density in the residual nucleus,

$$
P_{n}\left(\epsilon_{n}\right) d \epsilon_{n} \propto T_{n}\left(\epsilon_{n}, l, j\right) \rho\left(A-1, Z, U_{f}, J_{f}, \pi_{f}\right) d \epsilon_{n},
$$

where the accessible states in the residual nucleus are characterized by their energy $U_{f}=U_{i}-\epsilon_{n}-B_{n}$, spin $J_{f}$ and parity $\pi_{f} . U_{i}$ is the initial excitation energy. The neutron transmission coefficients are obtained by calculating the scattering matrix $S$ for the neutroninduced reaction on the residual fragment. By default, the global spherical optical model potential of Koning and Delaroche [6] is used in CGMF. Many fragments are deformed in their ground state, and more realistic potentials would be preferable. However, the global nature of the Koning-Delaroche potential makes it very suitable for such large-scale calculations. The spin and parity selection rules are applied at each stage of the decay, constraining the possible values of $J_{f}$ and $\pi_{f}$.

Similarly, the prompt $\gamma$-ray emission probability is equal to the product of the $\gamma$-ray transmission coefficients

(c) The Authors, published by EDP Sciences. This is an Open Access article distributed under the terms of the Creative Commons Attribution License 4.0 (http://creativecommons.org/licenses/by/4.0/). 
and the level density in the same fragment but with an excitation energy lowered by the energy of the emitted $\gamma$ ray,

$$
P_{\gamma}\left(\epsilon_{\gamma}\right) d \epsilon_{\gamma} \propto T_{\gamma}\left(\epsilon_{\gamma}\right) \rho\left(A, Z, U_{f}, J_{f}, \pi_{f}\right) d \epsilon_{\gamma},
$$

with $U_{f}=U_{i}-\epsilon_{\gamma}$. The $\gamma$-ray transmission coefficients are proportional to the strength function for each multipolarity considered. In this work, $E 1, M 1$ and $E 2$ transitions are considered.

The level density appearing in the equations above is a shortcut notation for describing a set of discrete levels at the lowest excitation energies followed by a continuum of levels above a certain matching energy. The discrete levels are taken from the RIPL-3 database [7] and the Gilbert-Cameron formalism is used to represent the continuum levels, with parameters also taken from RIPL-3. Some of the discrete states present in fission products are isomeric states with relatively long half-lives, whose decay will therefore produce a long tail in the time-dependent emission of "prompt" $\gamma$ rays. CGMF calculations have been shown to reproduce the observed time evolution of the $\gamma$ spectrum quite well [8]. At this point, the emission of both neutrons and $\gamma$ rays is assumed to be isotropic in the center-of-mass frame of the fission fragments. The kinematic boost of the neutrons lead to a strong focusing of the neutrons near the fission axis, as will be shown below.

\section{Numerical results}

The results of a CGMF run consists in a history file recording data for a large number of fission events. First, the fission fragment mass, charge, kinetic energy, and initial excitation energy, spin and parity are provided. Then, the neutron and $\gamma$-ray multiplicities are given, followed by the kinetic energy and directional cosines (in the laboratory frame) of each neutron and $\gamma$ ray emitted by this particular fragment. Then the same information is given for the complementary fragment. Such record is repeated a large number of times for as many fission events as one is interested in. Typically a few tens of thousand of events are needed to obtained a properly converged result for $\bar{v}$. The neutron multiplicity distribution $\mathrm{P}(v)$ requires a few hundred thousand events. But many millions of events are typically required for accurately describing fragment-mass-dependent PFNS. Prompt $\gamma$-ray data usually converge faster due to the larger number of emitted $\gamma$ rays compared to neutrons.

The average neutron multiplicity $\bar{v}$ and its distribution $\mathrm{P}(v)$ have been measured very precisely and consistently for ${ }^{252} \mathrm{Cf}$ (sf), as shown in Fig. 1 in comparison with CGMF results. The error bars on the evaluated data points [9] are actually smaller than the size of the points shown in Fig. 1. Although the value for $\bar{v}$ is very well reproduced by the calculation, the higher moments of the distribution deviate from the measured values. Those moments are actually very important for the interpretation of nuclear assays and neutron counting applications, and we are working to improve this agreement.

The calculated average multiplicity-dependent PFNS $\left\langle\chi_{\nu}\right\rangle$ are shown in Fig. 2 for $v=1 . .5$. We observe that the higher the multiplicity the harder the spectrum. As we know very well, the neutron multiplicity increases with increasing excitation energy, i.e. with decreasing total

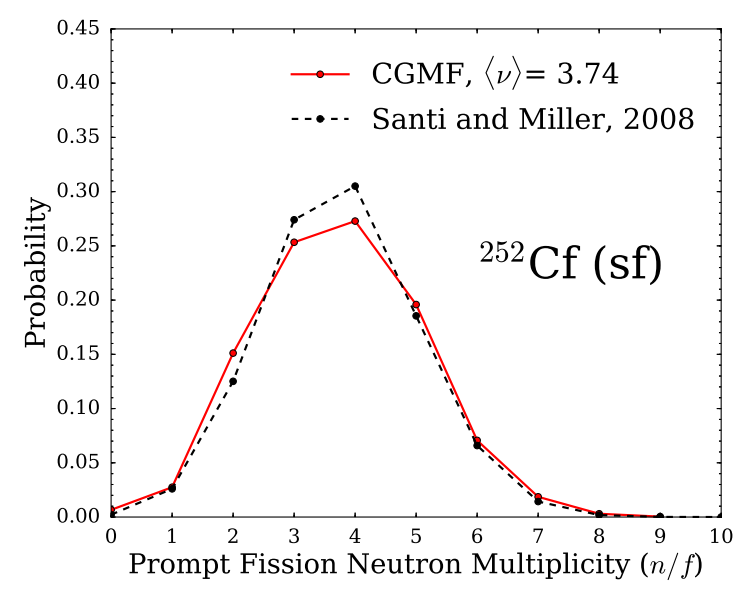

Figure 1. The prompt fission neutron multiplicity distribution $\mathrm{P}(v)$ calculated by CGMF is compared with the evaluation of Santi and Miller [9].

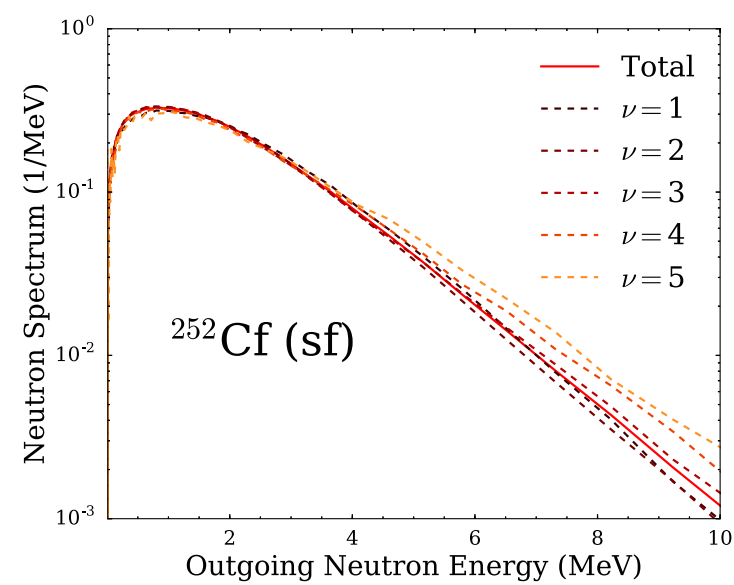

Figure 2. Calculated neutron multiplicity-dependent prompt fission neutron spectra show a harder spectrum with increasing neutron multiplicity.

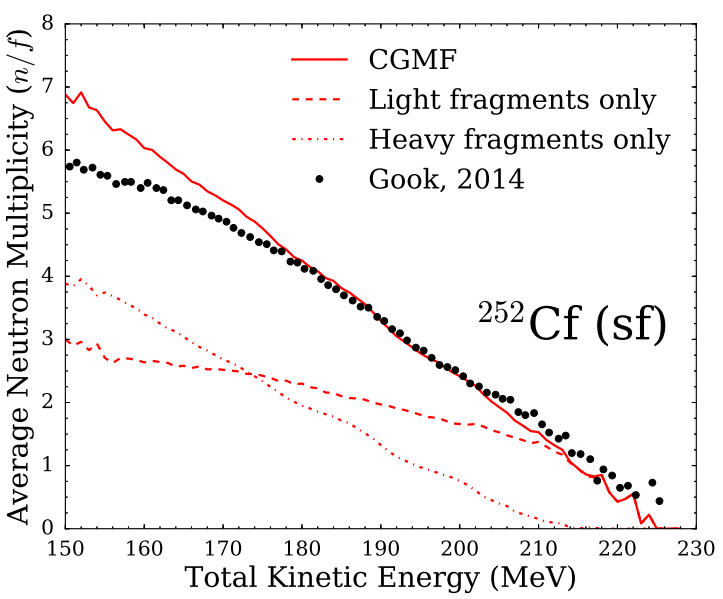

Figure 3. The average multiplicity is shown as a function of the total kinetic energy of the fragments.

kinetic energy. This plot shows that the extra excitation energy does not just go into increasing the number of emitted neutrons, but also indicates that the emitted neutrons are more energetic.

Figure 3 shows the variations of $\bar{v}$ with TKE, in comparison with Göök et al. data [11]. The agreement between calculated results and experimental data is 


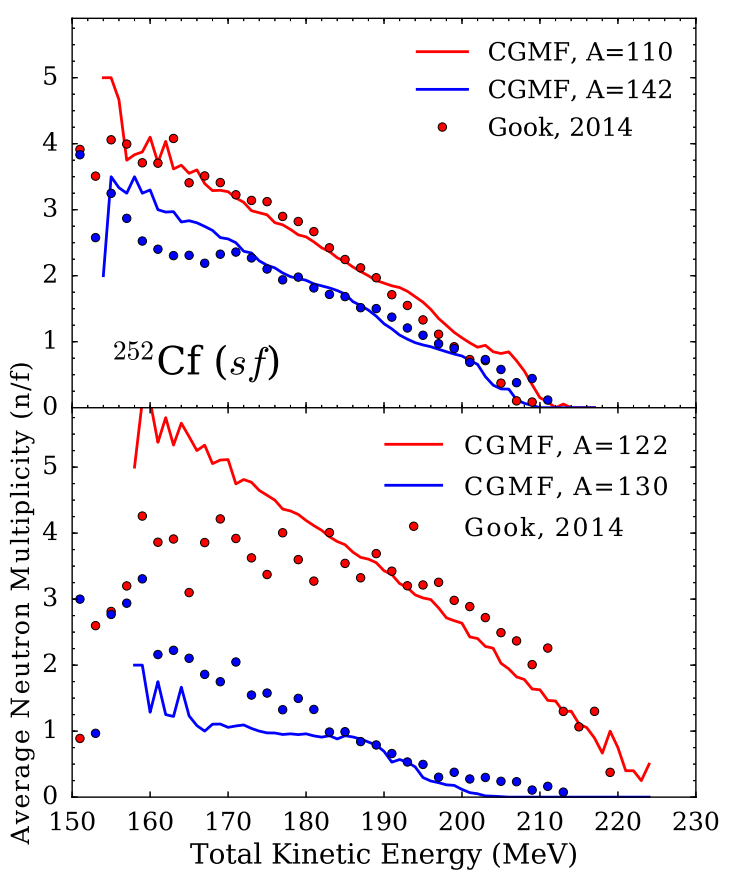

Figure 4. The average multiplicity is shown as a function of the total kinetic energy of the fragments, for two sets of fragment pairs.

reasonable, showing an almost linear decrease of $\bar{v}$ with TKE. Also shown are the separated components from the light and heavy fragments. For the highest TKE values, only the light fragments contribute and emit neutrons. While the curve for the heavy fragments is almost perfectly linear, the light fragment curve is not.

Of further interest is the decomposition of this result per mass split, as shown in Fig. 4 in comparison with Göök et al. data [11]. The agreement is quite reasonable in both pairs of fragments considered here, $A_{l} / A_{h}=110 / 142$ and $122 / 130$, and typical of other mass split results, showing that the neutron emission rates from different fragments is rather well reproduced by CGMF.

In CGMF, the neutrons are assumed to be emitted isotropically in the center-of-mass frame of the fully accelerated fragments. The kinematic boost into the laboratory tends to focus the neutrons at either 0 or 180 degrees with respect to the direction of emission of the light fragments, as measured by the angle $\theta_{n-L F}$. The distribution of $\theta_{n-L F}$ calculated by CGMF is shown in Fig. 5 in comparison with experimental data by Skarsvag et al. [12] and Bowman et al. [13]. The experimental and calculated results are in excellent agreement.

Neutron-neutron angular correlations have been measured by several groups in recent years. Figure 6 shows the $n-n$ angular correlations measured by Gagarski et al. [14] for different neutron energy thresholds from $425 \mathrm{keV}$ up to $1.6 \mathrm{MeV}$, and compared with CGMF results. The agreement is reasonable although there is a distinct bias as a function of the energy threshold. Note that the simulated results have not been folded with the detector response, which would be really important here. As expected, the neutron doubles are focused at 0 and 180 degrees. In the first case, the two neutrons are emitted from the same fragment, while in the latter scenario, they originate from the two complementary fragments.

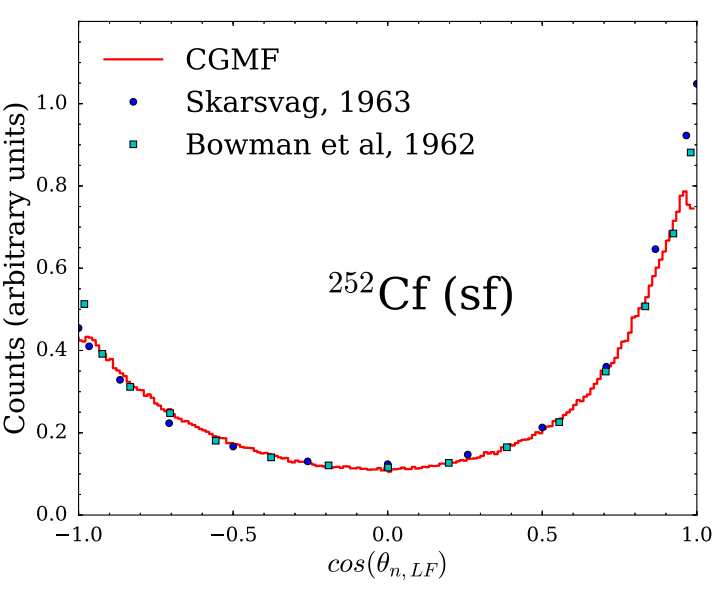

Figure 5. Prompt neutron angular distribution with respect to the direction of the light fragment. Experimental data sets are from Refs. [12,13].

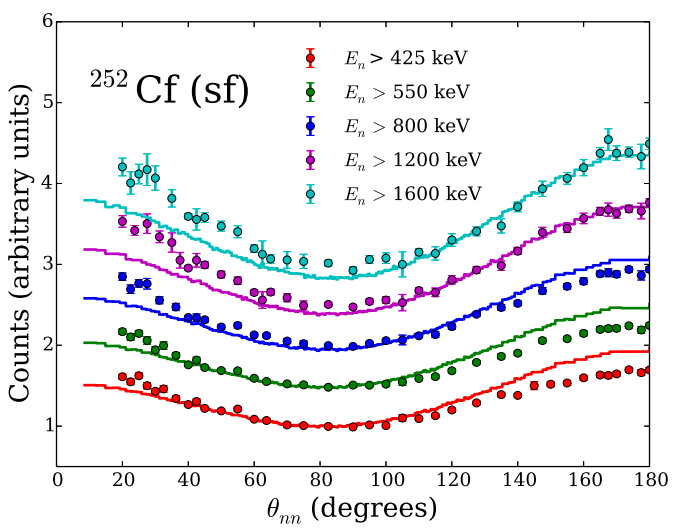

Figure 6. Neutron-neutron angular distributions as a function of a neutron energy threshold, as measured by Gagarski et al. [14], and calculated by CGMF.

As a final example, the time evolution of the cumulative average prompt $\gamma$-ray multiplicity, $\left\langle N_{\gamma}\right\rangle(t)$ is shown in Fig. 7. Fission fragments, before any $\beta$-decay takes place, can be formed in isomeric states that have half-lives ranging from nanoseconds to microseconds. The presence of these isomers delays the emission of some prompt $\gamma$ rays, leading to a monotonically increasing $\left\langle N_{\gamma}\right\rangle(t)$, as discussed at more length in [8].

\section{Integration with MCNP6.2}

The CGMF and FREYA [2] fission event generators are being integrated into the MCNP ${ }^{\circledR}$ transport code into its latest revision, MCNP6.2 [15]. This is a major breakthrough and a significant departure from the traditional approach that MCNP uses to transport neutrons and photons. Traditionally, MCNP does not follow the emissions on an event-by-event basis and uses instead clever weighting functions to properly describe reactions on average. This is particularly useful for criticality calculations where the reconstruction of each event is not required. However, some applications, such as non-proliferation, nuclear assays, detector development, require a more exact treatment and reconstruction of the reaction events to infer correlated signatures. The 6.2 version of MCNP is slated to be distributed before the end of this calendar year, and will come with both CGMF and FREYA options, along with 


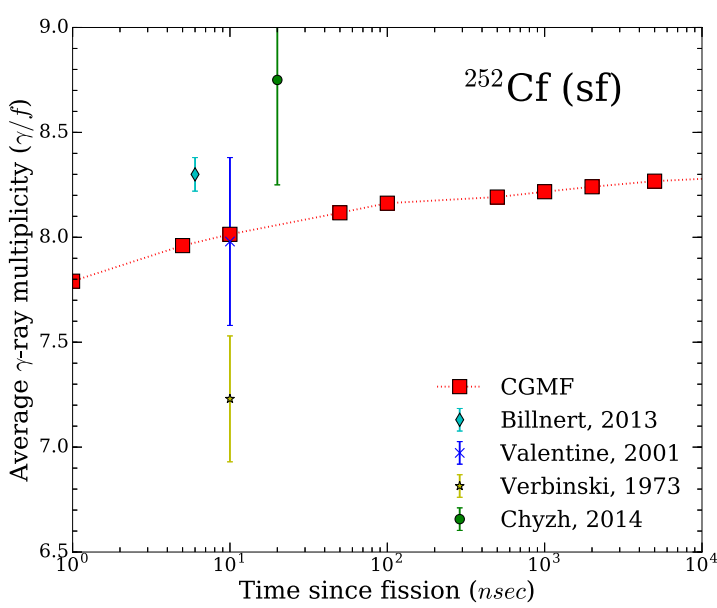

Figure 7. Cumulative average total prompt $\gamma$-ray energy as a function of time since fission. The calculated CGMF results are compared with several experimental data sets that each considered different time-coincidence windows.

a suite of tools that enable a more straightforward and efficient analysis of the list-mode PTRAC data.

\section{Conclusions}

The advent of fission event generators such as Monte Carlo Hauser-Feshbach codes is providing an access to nuclear data not available with simpler physics models. Exclusive data are very useful to challenge our current understanding of the fission process, while placing stringent constraints on important evaluated quantities such as PFNS, even in the case where only measurements of auxiliary prompt neutron observables exist. Prompt fission $\gamma$-ray characteristics are also computed consistently. New evaluations are in progress and will be incorporated in the B-VIII release of the U.S. ENDF/B library. Finally, the integration of such fission event generators into the MCNP6.2 transport code provides an important new tool for numerous applications. While only results for ${ }^{252} \mathrm{Cf}$ spontaneous fission were discussed in this paper, note that CGMF is applicable to various other spontaneous fission and neutron-induced fission reactions up to $20 \mathrm{MeV}$ incident neutron energy. More details can be found in the CGMF user manual [5].

The authors would like to thank A. Göök, F.-J. Hambsch, S. and A. Oberstedt, and A. Gatera for providing and discussing their experimental data. This work was performed at Los Alamos National Laboratory, under the auspices of the National Nuclear Security Administration of the U.S. Department of Energy at LANL under Contract No. DE-AC52-06NA25396. This work was partly supported by the Office of Defense Nuclear Nonproliferation Research \& Development (DNN R\&D), NNSA, US DOE.

\section{References}

[1] P. Talou et al., Phys. Rev. C 83, 064612 (2011)

[2] J.M. Verbeke, J. Randrup, and R. Vogt, Comp. Phys. Comm. 191, 178 (2015)

[3] O. Litaize, O. Serot, Phys. Rev. C 82, 054616 (2010)

[4] K.-H. Schmidt et al., Nucl. Data Sheets 131, 107 (2016)

[5] P. Talou, T. Kawano, I. Stetcu, CGMF User Manual, Los Alamos Technical Report, LA-UR-14-24031

[6] A. Koning, J.-P. Delaroche, Nucl. Phys. A713, 231 (2003)

[7] R. Capote et al., Nucl. Data Sheets 110, 3107 (2009)

[8] P. Talou et al., submitted to Phys. Rev. C

[9] P. Santi, M. Miller, Nucl. Sci. Eng. 160, 190 (2008)

[10] A. Carlson et al., Nucl. Data Sheets 100, 3215 (2009)

[11] A. Göök et al., Phys. Rev. C 90, 064611 (2014)

[12] K. Skarsvåg, K. Bergheim, Nucl. Phys. 45, 72 (1963)

[13] H.R. Bowman et al., Phys. Rev. 126, 2120 (1962)

[14] A. M. Gagarski et al., Bulletin of the Russian Academy of Sciences: Physics 72, 773 (2008)

[15] Initial MCNP6 Release Overview - MCNP6 version 1.0, MCNP6 Development Team, Los Alamos Technical Report, LA-UR-13-22934 (2013) 